\title{
Hamiltonian of mean force for damped quantum systems
}

\author{
Stefanie Hilt, Benedikt Thomas, and Eric Lutz \\ Department of Physics, University of Augsburg, 86135 Augsburg, Germany
}

(Dated: September 18, 2018)

\begin{abstract}
We consider a quantum system linearly coupled to a reservoir of harmonic oscillators. For finite coupling strengths, the stationary distribution of the damped system is not of the Gibbs form, in contrast to standard thermodynamics. With the help of the quantum Hamiltonian of mean force, we quantify this deviation exactly for a harmonic oscillator and provide approximations in the limit of high and low temperatures, and weak and strong couplings. Moreover, in the semiclassical regime, we use the quantum Smoluchowski equation to obtain results valid for any potential. We, finally, give a physical interpretation of the deviation in terms of the initial system-reservoir coupling.
\end{abstract}

PACS numbers: 03.67.-a, 05.30.-d

\section{INTRODUCTION}

Thermodynamics successfully describes equilibrium properties of macroscopic objects coupled to a heat bath. In the standard theory, the system-bath interaction is assumed to be small and is therefore neglected. This is a well-justified assumption when the coupling energy is small compared to the thermal energy of the system [1]. However, at low temperatures, quantum effects come into play and the interaction energy cannot be discarded anymore [2]. This is particularly relevant for solid-state nanodevices, such as nanomechanical oscillators, which may be strongly coupled to their environment [3]. In the limit of vanishingly small coupling, the damped quantum system asymptotically relaxes to the correct thermal Gibbs state [9]. However, for any finite interaction strength, the stationary state of the quantum system deviates from a Gibbs state, in contrast to the prediction of thermodynamics. It has, for example, been shown that, at zero temperature, the coupled oscillator is in an excited, mixed state and not in its pure ground state 10 12. In addition, it is worth noticing that the deviation from a Gibbs state leads to the interesting possibility of entangling two noninteracting coherent states via the indirect coupling to a common reservoir [13].

A useful quantity to describe classical systems that are strongly coupled to their environment is the so-called potential of mean force [14. It was first introduced by Kirkwood in his study of the average structure of liquids [15]. It has since proved to be an invaluable tool in chemistry [16], where it is commonly employed in the investigation of implicit solvent models 17 and of protein-ligand binding [18, to name a few examples. A quantum extension in the form of the Hamiltonian of mean force (HMF), see Eq. (5) below, has recently been proposed in Ref. [19]. Potential and Hamiltonian of mean force are instrumental in characterizing the deviations from standard thermodynamics that occur in the strong coupling regime. However, they are difficult to evaluate explicitly in the general case. Here, we use the quantum HMF to quantify the deviation from the thermal Gibbs state for a damped quantum harmonic oscillator, for which we obtain exact analytical results. We further derive approximate expres- sions for arbitrary systems in the strong damping regime. We additionally relate the HMF to the thermodynamic change of energy and entropy associated with the initial coupling between system and bath.

The present article is organized as follows. In Section II. we introduce the concept of the quantum HMF and show how it can be used to characterize the deviation from a thermal Gibbs state. In Section III, we determine the exact expression for this deviation for the case of the analytically solvable damped quantum harmonic oscillator. In Section IV, we provide useful approximations in the limit of high and low temperatures, weak and strong coupling. Furthermore in the semiclassical limit, we employ the quantum Smoluchowski equation to evaluate the deviation from the Gibbs state for any damped quantum system. In Section VI, we finally give a physical interpretation of the HMF by establishing a relationship with the initial system-bath coupling.

\section{HAMILTONIAN OF MEAN FORCE}

We consider the total Hamiltonian,

$$
H=H_{S}+H_{B}+H_{S B},
$$

which describes a quantum system $S$ coupled to a heat bath $B$ via the interaction $S B$. The equilibrium state of the total system is given by the Gibbs state,

$$
\rho=\frac{\exp (-\beta H)}{Z},
$$

where $Z=\operatorname{tr}\{\exp (-\beta H)\}$ is the total partition function and $\beta=1 /(k T)$ the inverse temperature of the bath. When the interaction energy is negligible, $H \simeq H_{S}+H_{B}$, the reduced density operator of the system, $\rho_{S}=\operatorname{tr}_{B}\{\rho\}$, is also of the Gibbs form,

$$
\rho_{S}=\frac{\exp \left(-\beta H_{S}\right)}{Z_{S}},
$$

with $Z_{S}=\operatorname{tr}_{S}\left\{\exp \left(-\beta H_{S}\right)\right\}$. However, for finite coupling, the interaction term cannot be neglected, and the 
reduced density operator is no longer of the Gibbs form. It can generically be written as,

$$
\rho_{S}=\frac{\exp \left(-\beta H_{S}^{*}\right)}{Z^{*}},
$$

where the quantum HMF is defined as the effective Hamiltonian 19 ,

$$
H_{S}^{*}=-\frac{1}{\beta} \ln \frac{\operatorname{tr}_{B}\{\exp (-\beta H)\}}{\operatorname{tr}_{B}\left\{\exp \left(-\beta H_{B}\right)\right\}} .
$$

The corresponding partition function is given by $Z^{*}=$ $\operatorname{tr}_{S}\left\{\exp \left(-\beta H_{S}^{*}\right)\right\}$. The HMF reduces to the Hamiltonian of the system, $H_{S}^{*} \simeq H_{S}$, in the limit of vanishing coupling. It is thus convenient to introduce the difference,

$$
\Delta H_{S}=H_{S}^{*}-H_{S} .
$$

The latter quantifies the deviation from a thermal Gibbs state. In the following, we evaluate the deviation $\Delta H_{S}$ explicitly for the damped quantum harmonic oscillator.

\section{MICROSCOPIC SYSTEM-RESERVOIR MODEL}

The standard model for a damped quantum harmonic oscillator is given by Hamiltonian (1) 20, where

$$
H_{S}=\frac{p^{2}}{2 M}+\frac{M}{2} \omega^{2} q^{2}
$$

is the Hamiltonian of the harmonic oscillator with mass $M$ and frequency $\omega$,

$$
H_{B}=\sum_{j=1}^{N}\left[\frac{p_{j}^{2}}{2 m_{j}}+\frac{m_{j} \omega_{j}^{2}}{2} x_{j}^{2}\right]
$$

describes the bath of $N$ harmonic oscillators, and

$$
H_{S B}=\sum_{j=1}^{N}\left[-C_{j} q x_{j}+\frac{C_{j}^{2}}{2 m_{j} \omega_{j}^{2}} q^{2}\right]
$$

is the coupling term linear in the position of the system. The frequencies of the reservoir modes are taken to be equidistant, that is $\omega_{j}=j \Delta$. The coupling constants $C_{j}$ are chosen to obey a Drude-Ullersma spectrum [20,

$$
C_{j}=\sqrt{\frac{2 \gamma m_{j} M \omega_{j}^{2} \Delta}{\pi} \frac{\omega_{D}^{2}}{\omega_{D}^{2}+\omega_{j}^{2}}},
$$

with damping coefficient $\gamma$ and cutoff frequency $\omega_{D}$. In the continuous limit $N \rightarrow \infty(\Delta \rightarrow 0)$, the bath is characterized by the Ohmic spectral density function [20],

$$
J(\nu)=\frac{\pi}{2} \sum_{j} \frac{C_{j}^{2}}{m_{j} \omega_{j}} \delta\left(\nu-\omega_{j}\right)=\frac{\gamma \nu M \omega_{D}^{2}}{\nu^{2}+\omega_{D}^{2}} .
$$

Due to the linearity of the model, the dynamics of the reduced quantum system is exactly solvable. Its stationary state is conveniently described in phase space using the Wigner function 21,

$W(q, p, t)=\int_{-\infty}^{\infty} \frac{d \xi}{2 \pi \hbar} \exp \left[-\frac{\mathrm{i}}{\hbar} p \xi\right] \rho_{S}\left(q+\frac{\xi}{2}, q-\frac{\xi}{2}, t\right)$,

where $\rho_{S}\left(x, x^{\prime}, t\right)=\left\langle x\left|\rho_{S}(t)\right| x^{\prime}\right\rangle$ is the position representation of the reduced density operator. The Wigner function of a damped harmonic oscillator is found to satisfy the exact quantum master equation 22,

$$
\begin{aligned}
\frac{\partial}{\partial t} W(q, p, t)= & -\frac{p}{M} \frac{\partial}{\partial q} W+\frac{\partial}{\partial p}\left[\left(\gamma p+M \omega^{2} q\right) W\right] \\
& +D_{p p}(t) \frac{\partial^{2}}{\partial p^{2}} W+D_{q p}(t) \frac{\partial^{2}}{\partial q \partial p} W .
\end{aligned}
$$

The detailed expressions for the diffusion coefficients $D_{p p}$ and $D_{q p}$ can be found in Ref. 22. The reduced stationary phase space distribution for the Ohmic model is given by the Gaussian Wigner function,

$$
W(q, p)=\frac{1}{2 \pi \sqrt{\left\langle q^{2}\right\rangle\left\langle p^{2}\right\rangle}} \exp \left[-\frac{q^{2}}{2\left\langle q^{2}\right\rangle}-\frac{p^{2}}{2\left\langle p^{2}\right\rangle}\right],
$$

with exact position and momentum dispersions [23],

$$
\begin{aligned}
\left\langle q^{2}\right\rangle & =\frac{\hbar}{M \pi} \sum_{j=1}^{3}\left[\frac{\left(\lambda_{j}-\omega_{D}\right) \psi\left(1+\frac{\beta \hbar \lambda_{j}}{2 \pi}\right)}{\left(\lambda_{j+1}-\lambda_{j}\right)\left(\lambda_{j-1}-\lambda_{j}\right)}\right] \\
& +\frac{1}{M \beta \omega^{2}}, \\
\left\langle p^{2}\right\rangle & =\frac{\hbar M \gamma \omega_{D}}{\pi} \sum_{j=1}^{3}\left[\frac{\lambda_{j} \psi\left(1+\frac{\beta \hbar \lambda_{j}}{2 \pi}\right)}{\left(\lambda_{j+1}-\lambda_{j}\right)\left(\lambda_{j-1}-\lambda_{j}\right)}\right] \\
& +M^{2} \omega^{2}\left\langle q^{2}\right\rangle(\eta, M) .
\end{aligned}
$$

The parameters $\lambda_{i}$ are the characteristic frequencies of the damped oscillator and $\psi$ is the digamma function. In the limit of large cutoff frequencies, $\omega_{D} \gg \gamma, \omega$, the characteristic frequencies can be approximated by [23],

$$
\begin{aligned}
\lambda_{1,2} & \simeq \frac{\gamma}{2} \pm \sqrt{\frac{\gamma^{2}}{4}-\omega^{2}}, \\
\lambda_{3} & =\omega_{D}-\gamma .
\end{aligned}
$$

A direct consequence of Eq. 16 is that equipartition is in general violated, $M \omega^{2}\left\langle q^{2}\right\rangle<\left\langle p^{2}\right\rangle / M$, and $\rho_{S}$ is not of the Gibbs form. This can be understood by noting that due to the form of the coupling Eq. (9), the bath can be seen as continuously measuring the position of the system 20]. As a result, the variance of the position is reduced. The momentum dispersion then increases following the Heisenberg uncertainty relation. For a vanishingly coupled oscillator, the variances reduce to

$$
M \omega^{2}\left\langle q^{2}\right\rangle_{\gamma=0}=\frac{\left\langle p^{2}\right\rangle_{\gamma=0}}{M}=\frac{\hbar \omega}{2} \operatorname{coth}\left(\frac{\beta \hbar \omega}{2}\right) .
$$


Equipartition therefore holds and the reduced stationary state is Gibbsian. The position representation of the reduced density operator is further given by [20],

$$
\rho_{S}\left(q, q^{\prime}\right)=\frac{1}{\sqrt{2 \pi\left\langle q^{2}\right\rangle}} \exp \left[-\frac{\left(q+q^{\prime}\right)^{2}}{8\left\langle q^{2}\right\rangle}-\frac{\left(q-q^{\prime}\right)^{2}}{2 \hbar^{2} /\left\langle p^{2}\right\rangle}\right] .
$$

\section{HMF FOR DAMPED QUANTUM OSCILLATOR}

The exact expression for the quantum HMF (5) for the damped oscillator can be obtained in the following way 23. Since the stationary state Eq. (14) is a Gaussian, it can be regarded as the equilibrium Wigner function of an effective harmonic oscillator with Hamiltonian,

$$
H_{S}^{*}=\frac{p^{2}}{2 M^{*}}+\frac{1}{2} M^{*} \omega^{* 2} q^{2},
$$

with mass $M^{*}$ and frequency $\omega^{*}$. The latter reads 24,

$$
W(q, p)=\frac{\tanh \left(\beta \hbar \omega^{*} / 2\right)}{\pi \hbar} \exp \left[\frac{2 H_{S}^{*}}{\hbar \omega^{*} \operatorname{coth}\left(\beta \hbar \omega^{*} / 2\right)}\right] .
$$

By comparing the two Wigner functions Eq. (14) and Eq. (21), one can identify the effective mass,

$$
M^{*}=\sqrt{\frac{\left\langle p^{2}\right\rangle}{\left\langle q^{2}\right\rangle}} \frac{\beta \hbar}{2} \frac{1}{\operatorname{arcoth}(2 v)},
$$

and the effective frequency,

$$
\omega^{*}=\frac{2}{\beta \hbar} \operatorname{arcoth}(2 \nu) .
$$

Here $v=\sqrt{\left\langle p^{2}\right\rangle\left\langle q^{2}\right\rangle} / \hbar$ is the phase space volume. In order to estimate the effect of the finite coupling on the reduced state of the system, we now determine the deviation $\Delta H_{S}$ given by Eq. (6). Combining Eqs. 20), (22) and 23 for the quantum $\mathrm{HMF}$, we arrive at

$$
\Delta H_{S}=A \frac{p^{2}}{2}+B \frac{q^{2}}{2},
$$

where we have introduced the two coefficients

$$
\begin{aligned}
A & =\sqrt{\frac{\left\langle q^{2}\right\rangle}{\left\langle p^{2}\right\rangle}} \frac{2}{\beta \hbar} \operatorname{arcoth}(2 v)-\frac{1}{M}, \\
B & =\sqrt{\frac{\left\langle p^{2}\right\rangle}{\left\langle q^{2}\right\rangle}} \frac{2}{\beta \hbar} \operatorname{arcoth}(2 v)-M \omega^{2} .
\end{aligned}
$$

Equation 24 gives the exact expression for deviation $\Delta H_{S}$ for the damped quantum oscillator. The coefficients $A$ and $B$ are divergent for $2 v=1, \operatorname{since} \operatorname{arcoth} x$ is not defined at $x=1$. This happens for an undamped

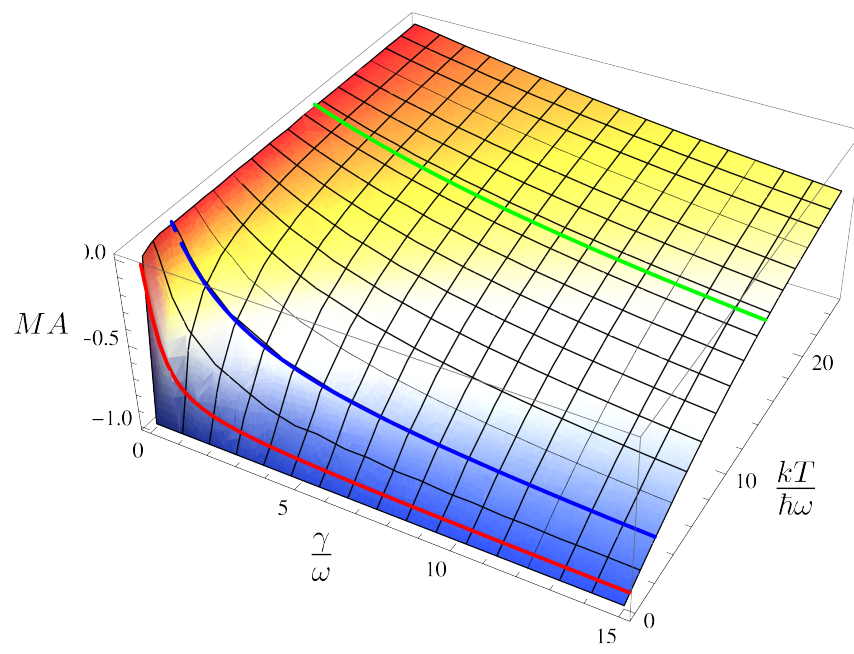

(a)

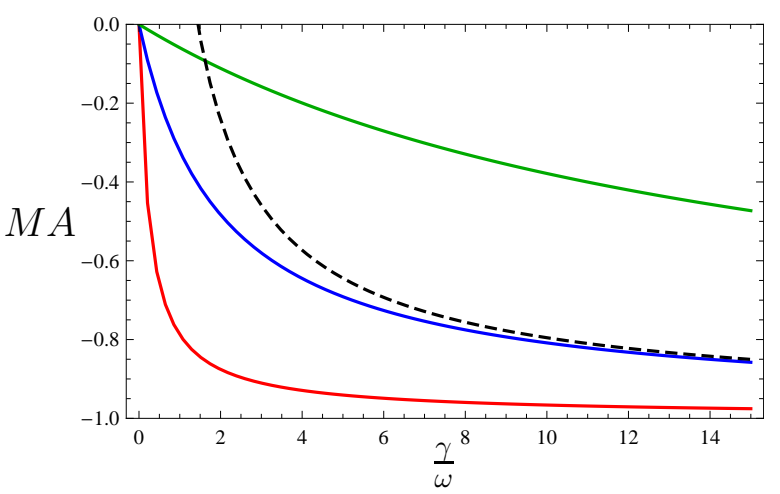

(b)

FIG. 1: (Color online) (a) Coefficient $M A$, Eq. (25), as a function of the dimensionless parameters $k T /(\hbar \omega)$ and $\gamma / \omega$. $A$ increases monotonically to zero with temperature and inverse coupling strength. The deviation from a Gibbs state in momentum is thus maximal in the strong-coupling, lowtemperature limit. (b) Cross sections of the $(T, \gamma)$-curve for $k T /(\hbar \omega)=0.5$ (red lower line), 3 (blue line), 15 (green upper line). In the limit of high damping, $A$ coincides with the semiclassical expression given by the quantum Smoluchowski equation (black dashed line for $k T /(\hbar \omega)=3$ ), Eq. (37). Parameters are $\omega_{D}=1000, \omega=1, M=1$.

harmonic oscillator $(\gamma=0)$ in its ground state $(T=0)$. However, in this case $A=B=0$, as the system relaxes to a Gibbs state.

Figure 1 shows the coefficient $A$, Eq. (25), as a function of temperature and coupling strength. $A$ is always negative, since $-1 \leq M A \leq 0$. We observe that $A$ decreases monotonically with the coupling and the inverse temperature. The maximum deviation from the Gibbs state is thus achieved in the low-temperature, strong coupling limit. Moreover, for weak coupling, $A$ vanishes rapidly with growing temperature, whereas it decays much more slowly in the limit of strong coupling, indicating that the deviation from a Gibbs state persists at much higher temperatures in the latter. The coeffi- 


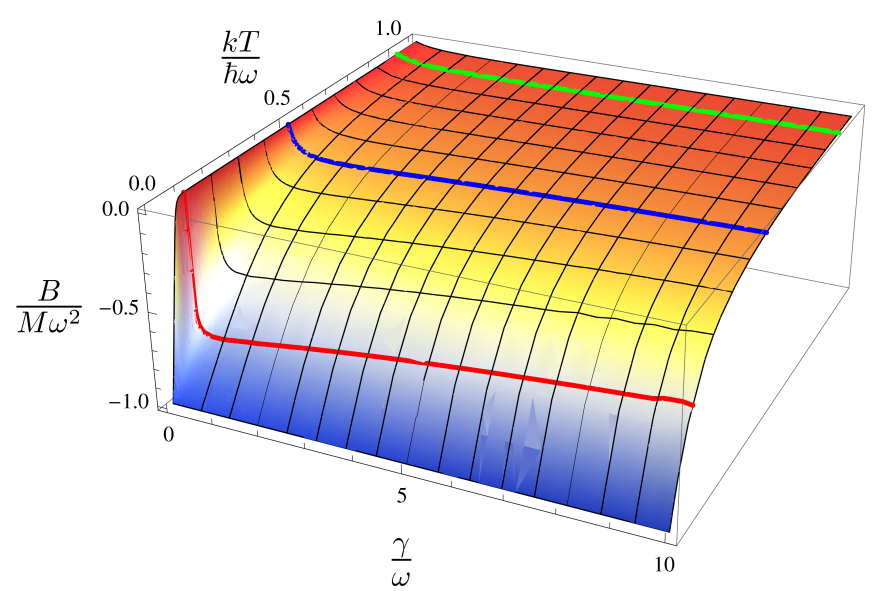

(a)

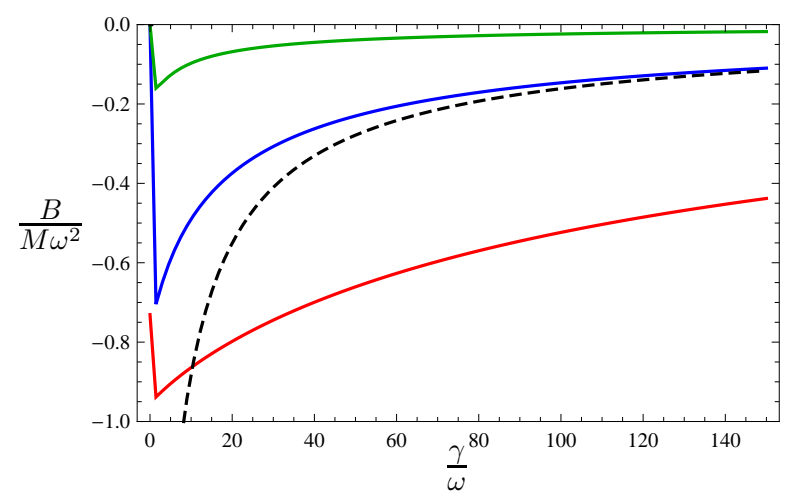

(b)

FIG. 2: (Color online) (a) Coefficient $B /\left(M \omega^{2}\right)$, Eq. 26), as a function of the dimensionless parameters $k T /(\hbar \omega)$ and $\gamma / \omega$. $B$ increases monotonically to zero with temperature. However, it exhibits a minimum as a function of the coupling strength. (b) Cross sections of the $(T, \gamma)$-curve for $k T /(\hbar \omega)=0.1$ (red lower line), 0.5 (blue line), 1 (green upper line). For increasing temperatures, the dip gets less pronounced and eventually disappears. In the limit of highdamping, $B$ coincides with the semiclassical expression given by the quantum Smoluchowski equation (black dashed line for $k T /(\hbar \omega)=0.1)$, Eq. 45. Same parameters as in Fig. 1 .

cient $B$, Eq. (26), is plotted in Fig. 2(a) as a function of temperature and coupling strength. It is also negative with $-1 \leq B /\left(M \omega^{2}\right) \leq 0$. While $B$ shows a similar temperature dependence as the coefficient $A$, its dependence on the coupling strength differs significantly. The three lines highlighted in Fig. 2(a), and reproduced for clarity in Fig. 2(b), show that $B$ possesses a minimum. Hence, the deviation of the Gibbs state in position increases up to a certain value of $\gamma$ before decreasing even though the coupling becomes larger. The presence of a minimum can be understood by noting that $\left\langle q^{2}\right\rangle$ decreases with increasing coupling constant, while $\left\langle p^{2}\right\rangle$ increases 20. As a result, the ratio $\left\langle p^{2}\right\rangle /\left\langle q^{2}\right\rangle$ increases faster than the product $\left\langle p^{2}\right\rangle\left\langle q^{2}\right\rangle$. The hyperbolic arccotangent of the phase space volume in Eq. (26), therefore, decreases for grow-

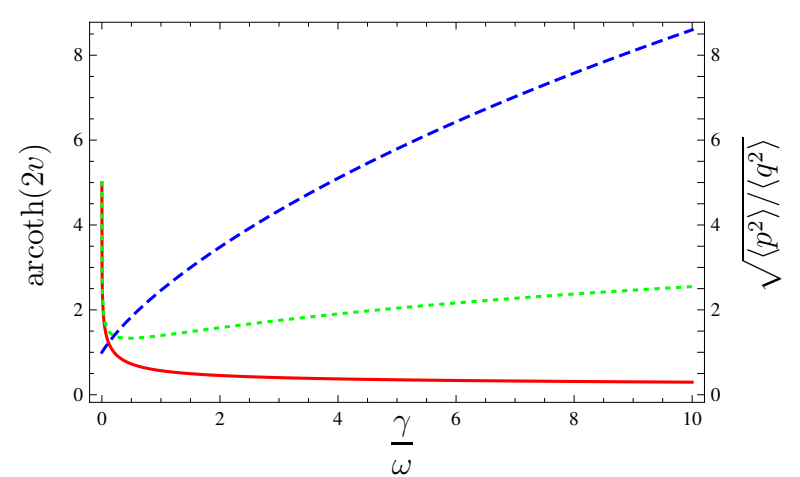

FIG. 3: (Color online) Function $\operatorname{arcoth}(2 v)$ (red continuous) and $\sqrt{\left\langle p^{2}\right\rangle /\left\langle q^{2}\right\rangle}$ (blue dashed) appearing in the definition of the coefficient $B$, Eq. 26, as a function of the dimensionless parameter $\gamma / \omega$. Their different behaviors lead to a minimum for their product (green dotted line). Same parameters as in Fig. 1 and $k T / \hbar \omega=0.1$.

ing coupling constants. Its product with the increasing square root in front thus displays a minimum (see Fig. 3). Figure 2(b) additionally indicates that the minimum gets less pronounced for higher temperatures and eventually disappears in the classical limit. Finally, in Fig. 4 , we show the average relative deviation $\left\langle\Delta H_{S}\right\rangle /\left\langle H_{S}\right\rangle$, which vanishes in the high-temperature limit. It is worth noticing that this behavior is peculiar to the linear coupling model that we consider here. For the case of nonlinear system-bath coupling, the deviation exists even in the classical limit 25 . Since $A\left\langle p^{2}\right\rangle$ is in general much larger than $B\left\langle q^{2}\right\rangle$ (see below), the effect we have observed for $B$ is suppressed, and the deviation decreases monotonically with increasing coupling strength. In the following, we derive analytic expressions for the coefficients $A$ and $B$ in various limits of interest, including the limits of low and high temperatures, weak and strong couplings. In all cases, we assume the cutoff frequency $\omega_{D}$ to be large.

\section{A. High-temperature limit}

In the high-temperature limit, $\hbar \omega \ll k T$, the position and momentum quadratures $(15)$ and $(16)$ can be expanded to lowest order to yield [23],

$$
\begin{aligned}
& \left\langle q^{2}\right\rangle \simeq \frac{k T}{M \omega^{2}}\left[1+\frac{1}{12}\left(\frac{\hbar \omega}{k T}\right)^{2}\right], \\
& \left\langle p^{2}\right\rangle \simeq M k T\left[1+\frac{1}{12} \frac{\hbar^{2}\left(\omega^{2}+\gamma \omega_{D}\right)}{(k T)^{2}}\right],
\end{aligned}
$$

where we have made use of the series expansion of the digamma function Eq. A2 . We note that the coupling constant $\gamma$ only appears in second order in $(\hbar \omega / k T)$. The two variances Eqs. 27) and (28), hence, become damping independent in the classical limit, and equipartition holds, $\left\langle p^{2}\right\rangle / M=M \omega^{2}\left\langle q^{2}\right\rangle=k T$. For high temperatures, the phase space volume $v$ is large and the arcoth in the 


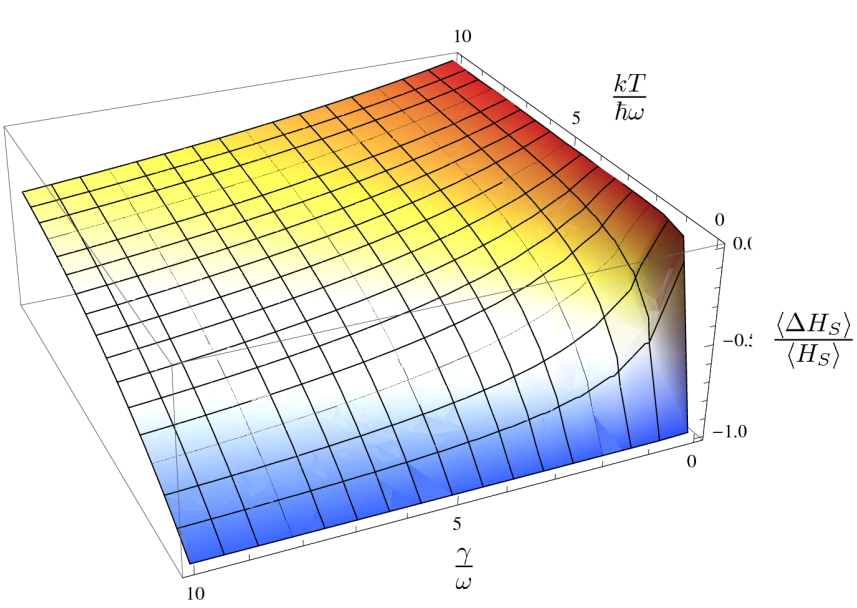

FIG. 4: (Color online) Relative mean deviation $\left\langle\Delta H_{S}\right\rangle /\left\langle H_{S}\right\rangle$, Eq. 24), showing the departure from a Gibbs state as a function of dimensionless temperature and coupling strength. The maximum deviation is observed in the low-temperature, strong-coupling regime. Same parameters as in Fig. 1]

coefficients $A$ and $B$, Eqs. 25) and (26), can be approximated with the help of Eq. (A3). We obtain,

$$
\begin{aligned}
& A \simeq-\frac{1}{12 M} \frac{\hbar^{2}\left(\omega^{2}+\gamma \omega_{D}\right)}{(k T)^{2}}, \\
& B \simeq-\frac{M \omega^{2}}{12}\left(\frac{\hbar \omega}{k T}\right)^{2} .
\end{aligned}
$$

Both coefficients approach zero quickly as $-1 / T^{2}$, see Figs. 1 and 2(a) However, unlike $B$, the coefficient $A$ depends explicitly on $\gamma$ and $\omega_{D}$. This feature follows from the Ohmic nature of the damping, as seen from Eq. 28 for $\left\langle p^{2}\right\rangle$. In the limit of high cutoff frequency, $A$ is much larger than $B$. Combining Eqs. $(27)-(30)$, we find that the total deviation $\Delta H_{S} \sim-1 / T^{2}$ in the classical limit, see Fig. 4. In this regime, the stationary state of the system reduces to a classical Gibbs distribution, $W(q, p)=\mathcal{N} \exp \left[-\left(M \omega^{2} q^{2}+p^{2} / M\right) /(2 k T)\right]$.

\section{B. Low-temperature limit}

For low temperatures, $k T \ll \hbar \omega$, we can approximate the digamma function in Eqs. (15) and (16) with the help of Eq. A1. To proceed further, it is important to distinguish between weak and strong coupling limit. For weak coupling, $k T \ll \hbar \gamma \ll \hbar \omega$, an expansion up to second order in $k T / \hbar \omega$ and first order in $\gamma / \omega$ yields [23,

$$
\begin{aligned}
& \left\langle q^{2}\right\rangle \simeq \frac{\hbar \omega}{2 M \omega^{2}}\left[1-\frac{\gamma}{\omega}\left(\frac{1}{\pi}-\frac{2 \pi}{3}\left(\frac{k T}{\hbar \omega}\right)^{2}\right)\right], \\
& \left\langle p^{2}\right\rangle \simeq \frac{\hbar \omega M}{2}\left[1-\frac{\gamma}{\omega}\left(\frac{1}{\pi}-\frac{2}{\pi} \ln \frac{\omega_{D}}{\omega}\right)\right] .
\end{aligned}
$$

For $\gamma=0$, Eqs. (31) and (32) lead to the correct ground state mean energy of an undamped harmonic oscillator, $\left\langle H_{S}\right\rangle=\left\langle p^{2}\right\rangle / 2 M+M \omega^{2}\left\langle q^{2}\right\rangle / 2=\hbar \omega / 2$. At the same time, the phase space volume can be approximated by $v=1 / 2(1+C)$, where $C=\gamma\left(\ln \left(\omega_{D} / \omega\right)-1\right) /(\omega \pi)$. Therefore, making use of Eq. (A4), we find the coefficients,

$$
\begin{aligned}
& A \simeq-\frac{1}{M}\left[\frac{k T}{\hbar \omega}(1-Y) \ln (C / 2)+1\right], \\
& B \simeq-M \omega^{2}\left[\frac{k T}{\hbar \omega}(1+Y) \ln (C / 2)+1\right],
\end{aligned}
$$

with $Y=\gamma \ln \left(\omega_{D} / \omega\right) /(\omega \pi)$. Here, in contrast to the high-temperature limit, both $A$ and $B$ increase with $T$. As a result, the deviation from the Gibbs state decreases with increasing temperature, see Figs. 1 and 2(a). At zero temperature, the coefficients $A$ and $B$ attain their minimum values, $A=-1 / M$ and $B=-M \omega^{2}$, that correspond to the maximum deviation from the Gibbs state.

In the opposite limit of strong damping, $k T \ll \hbar \omega \ll$ $\hbar \gamma$, a lowest order expansion of the variances, Eqs. 15 and 16 leads to 23 ,

$$
\begin{aligned}
& \left\langle q^{2}\right\rangle=\frac{2 \hbar}{\pi M \gamma} \ln \frac{\gamma}{\omega}+\frac{\pi \hbar \gamma}{3 M \omega^{2}}\left(\frac{k T}{\hbar \omega}\right)^{2}, \\
& \left\langle p^{2}\right\rangle=\frac{\hbar M \gamma}{\pi} \ln \frac{\omega_{D}}{\gamma},
\end{aligned}
$$

In this regime, the phase space volume $v$ is large and the arcoth in $A$ and $B$, Eqs. (25) and (26), can therefore be approximated by Eq. A3. We then obtain in first order,

$$
\begin{aligned}
A & =\frac{1}{M}\left[\frac{k T}{\hbar \omega} \frac{\omega}{\gamma} \frac{\pi}{\ln \left(\omega_{D} / \gamma\right)}-1\right] \\
B & =M \omega^{2}\left[\frac{\gamma}{\omega} \frac{k T}{\hbar \omega} \frac{\pi}{2 \ln (\gamma / \omega)}-1\right] .
\end{aligned}
$$

Again the two coefficients increase linearly with $T$, see Figs. 1 and 2(a)

\section{SEMICLASSICAL LIMIT}

In the overdamped regime, the dynamics of the quantum particle simplifies considerably. In the limit $\gamma / \omega^{2} \gg$ $(\hbar \beta, 1 / \gamma)$, the off-diagonal matrix elements of the reduced density operator of the system in the coordinate representation are strongly suppressed, and a semiclassical description becomes possible. On the coarsed-grained time scale, $t \gg 1 / \gamma$, the diffusion coefficients in the master equation (13) simplify to [26, 27,

$$
\begin{aligned}
& D_{q p}=V^{\prime \prime}(q)\left\langle q^{2}\right\rangle-\frac{\left\langle p^{2}\right\rangle}{M}, \\
& D_{p p}=\gamma\left\langle p^{2}\right\rangle .
\end{aligned}
$$

The position and momentum variances for the harmonic oscillator are given in this limit by 28,

$$
\begin{aligned}
\left\langle q^{2}\right\rangle & =\frac{1}{\beta M \omega^{2}}+\lambda, \\
\left\langle p^{2}\right\rangle & =\frac{\hbar M \gamma}{\pi} \ln \frac{\omega_{D}}{\gamma}=\Omega,
\end{aligned}
$$


where $\lambda=\hbar \log [\hbar \beta \gamma /(2 \pi)] /(\pi M \gamma)$ measures the strength of quantum fluctuations. The master equation (13) hence reduces to,

$$
\begin{array}{r}
\frac{\partial}{\partial t} W(q, t)=-\frac{p}{M} \frac{\partial}{\partial q} W+\frac{\partial}{\partial q}\left[V^{\prime}(q, t)+\gamma p\right] W \\
+\gamma\left\langle p^{2}\right\rangle \frac{\partial^{2}}{\partial p^{2}}+\frac{\partial^{2}}{\partial p \partial q}\left[\frac{D(q)}{\beta}-\frac{\left\langle p^{2}\right\rangle}{M}\right] W,
\end{array}
$$

with $D(q)=1+\beta \lambda V^{\prime \prime}(q)$. As discussed in Refs. 29, 30], the effective diffusion coefficient $D(q)$ should be regarded, for thermodynamic consistency, as the first order expansion of $D(q)=\left(1-\beta \lambda V^{\prime \prime}(q)\right)^{-1}$. Equation (43) has been derived for arbitrary potentials $V(q)$ in Ref. 31] (note the presence of the incorrect effective potential $V_{\text {eff }}$ in the latter [28]). The stationary solution of Eq. (43) reads,

$$
W(q, p)=N \exp \left[-\beta V(q)+\frac{\lambda \beta^{2}}{2} V^{\prime}(q)^{2}-\frac{p^{2}}{2 \Omega}\right] .
$$

For the special case of a harmonic potential, $V(q)=$ $M \omega^{2} q^{2} / 2$, we can immediately identify the coefficient,

$$
B=-\frac{M^{2} \omega^{4}}{k T} \lambda
$$

where we made use of Eq. (24). It is important to realize that the semiclassical equation $(43)$ is valid both in the high-temperature, $k T \gg \hbar \gamma$, and low-temperature, $k T \ll \hbar \gamma$, regimes. In the high-temperature limit $\lambda=$ $\hbar^{2} /(12 M k T)$ and Eq. (41) reduces to Eq. 27).

For general potentials $V(q)$, the deviation from the Gibbs state takes the form,

$$
\Delta H_{S}=A \frac{p^{2}}{2}+\frac{\lambda \beta}{2} V^{\prime}(q)^{2},
$$

where the coefficient $\mathrm{A}$ is given by,

$$
A=\frac{1}{\beta \Omega}-\frac{1}{M},
$$

and is independent of the system. In the semiclassical strong-coupling domain, the deviation from the Gibbs state can thus be determined for arbitrary potentials and not only for the harmonic oscillator. We mention that in the high-temperature limit, the semiclassical Hamiltonian of mean force has been examined for a free particle and a harmonic dumbbell up to order $\hbar^{2}$ in Ref. 25. We note, moreover, that the deviation in position can be directly obtained from the simpler quantum Smoluchowski equation which follows from the the semiclassical phasespace master equation (43) [27, 28, 31, 32],

$$
\frac{\partial}{\partial t} W(q, t)=\frac{1}{\gamma M} \frac{\partial}{\partial q}\left[V^{\prime}(q, t)+\frac{1}{\gamma} D(q)\right] W(q, t),
$$

where we have introduced the distribution,

$$
W(q, t)=\rho_{S}(q, q, t)=\int d p W(q, p, t),
$$

The corresponding stationary solution is

$$
W(q)=N_{q} \exp \left[-\beta V(q)+\frac{\lambda \beta^{2}}{2} V^{\prime}(q)^{2}\right],
$$

\section{PHYSICAL INTERPRETATION OF $\Delta H_{S}$}

A physical interpretation of the difference $\Delta H_{S}$ between the quantum HMF and the system Hamiltonian can be given by considering the initial coupling between system and bath. By treating the latter process as a thermodynamic transformation, both the change of energy and entropy of the quantum system during the coupling can be determined 33. We assume that system and bath are initially decoupled, and each in thermal equilibrium at temperature $T$. The total density operator is thus given by the direct product,

$$
\rho=\frac{\exp \left(-\beta H_{S}\right)}{Z_{S}} \otimes \frac{\exp \left(-\beta H_{B}\right)}{Z_{B}} .
$$

If the system-bath interaction is switched on quasistatically, the entropy of the system changes by $\Delta S$ and an amount $Q$ of heat is exchanged with the heat bath. Both can be evaluated by writing the reduced density operator of the system Eq. (4) in the form,

$$
\rho_{S}=\exp \left[-\beta\left(H_{S}+\Delta H_{S}-F\right)\right],
$$

where $F=-(1 / \beta) \ln Z^{*}$ is the free energy of the system. The von Neumann entropy of the coupled system, $S=$ $-k \operatorname{tr}_{S}\left\{\rho_{S} \ln \rho_{S}\right\}$, is then,

$$
T S=\left(U-F+\left\langle\Delta H_{S}\right\rangle\right),
$$

where $U=\left\langle H_{S}\right\rangle=\operatorname{tr}_{S}\left\{\rho_{S} H_{S}\right\}$ is the internal energy of the coupled system. According to the first law, the heat exchanged with the bath during the coupling process is $Q=\Delta U-W$, where the work is equal to the free energy difference between uncoupled and uncoupled system $W=\Delta F[12$. We, therefore, have,

$$
Q=k T \Delta S-\left\langle\Delta H_{S}\right\rangle,
$$

where we have made use of the fact that, before the coupling $(\gamma=0), U_{0}=k T S_{0}-F_{0}$. The above result, valid for any quantum dissipative system, shows that the average difference between the HMF and the bare Hamiltonian of the system is just the difference between $k T \Delta S$ and the heat exchange $Q$ during the coupling process. For vanishingly small coupling, we find $Q=k T \Delta S$, in agreement with standard thermodynamics.

\section{CONCLUSION}

For a damped quantum system deviations from standard thermodynamics occur in the finite coupling regime. With the help of the quantum HMF, we have derived an exact expression for the deviation from a thermal Gibbs state for a damped quantum harmonic oscillator coupled to an Ohmic heat bath. We have obtained useful approximations in the limit of high and low temperature, and of weak and strong coupling. In the semiclassical regime, we 
have, moreover, used the quantum Smoluchowski equation to derive an approximate formula for the HMF valid for any quantum system. Finally, we have established a connection between the deviation from a Gibbs state and the thermodynamic change of the system that occur during the initial coupling process. Our findings emphasize the importance of the HMF in the thermodynamic analysis of strong damped quantum systems.

This work was supported by the Emmy Noether Program of the DFG (contract No LU1382/1-1) and the cluster of excellence Nanosystems Initiative Munich (NIM).

\section{Appendix A}

We collect, for convenience, the series expansions of the digamma function used in the evaluation of the position and momentum quadratures, Eqs. (15) and (16), 34,

$$
\psi(x)=\ln x-\frac{1}{2 x}-\frac{1}{12 x^{2}}+\mathcal{O}\left(\frac{1}{x^{4}}\right) \quad|x|>1
$$

and

$$
\psi(1+x)=-C+\frac{\pi^{2}}{6} x+\mathcal{O}\left(x^{2}\right) \quad|x|<1
$$

where $C$ is the Euler constant. On the other hand, the series expansions of the hyperbolic cotangent, needed in the computation of the coefficients $A$ and $B$, Eqs. 25 and $(26)$, are 34,

$$
\operatorname{arcoth}(y)=\frac{1}{y}+\mathcal{O}\left(\frac{1}{y^{3}}\right) \quad|y|>1
$$

and

$$
\operatorname{arcoth}(1+y) \approx-\frac{1}{2} \ln \left(\frac{y}{2}\right) \quad|y|<1 .
$$

[1] R. Kubo, Thermodynamics, (North-Holland, Amsterdam, 1968).

[2] P. Hänggi and G.L. Ingold, Chaos 15, 026105 (2005).

[3] A.N. Cleland, Foundations of Nanomechanics, (Springer, Berlin, 2003).

[4] M. Roukes, Phys. World 14, 25 (2001); H.G. Craighead, Science 290, 1532 (2000)

[5] C. A. Regal, J. D. Teufel, and K. W. Lehnert, Nature Phys., 4, 555 (2008).

[6] R. G. Knobel and A. N. Cleland, Nature 424, 291-293 (2003).

[7] A.D. O'Connell et al., Nature 464, 697 (2010).

[8] I. Wilson-Rae, Phys. Rev. B 77, 245418 (2008)

[9] R. Benguria and M. Kac, Phys. Rev. Lett. 46, 1 (1981).

[10] K. Lindenberg and B.J. West, Phys. Rev. A 30, 568 (1984).

[11] X.L. Li, G.W. Ford, and R.F. O'Connell, Phys. Rev. E 51, 5169 (1995).

[12] G.W. Ford and R.F. O'Connell, Phys. Rev. Lett. 96, 020402 (2006).

[13] J.P. Paz and A. Roncaglia, Phys. Rev. Lett. 100, 220401 (2008).

[14] M.E. Tuckerman, Statistical Mechanics: Theory and Molecular Simulation, (Oxford, Oxford, 2010).

[15] J. G. Kirkwood, J. Chem. Phys. 3, 300 (1935).

[16] T.Hill, Statistical Mechanics, McGraw-Hill, New York, 1956.

[17] B. Roux and T. Simonson, Biophys. Chem. 78, 1 (1999).

[18] T. Rodinger, P. L. Howell, and R. Pomes, J. Chem. Phys. 129, 155102 (2008).

[19] M. Campisi, P. Talkner, and P. Hänggi, Phys. Rev. Lett. 102, 210401 (2009).
[20] U. Weiss, Quantum Dissipative Systems, (World Scientific, Singapore, 1999).

[21] W.P. Schleich, Quantum Optics in Phase Space, (Wiley, Berlin, 2001).

[22] B. L. Hu, J. P. Paz, and Y. Zhang, Phys. Rev. D 45, 2843 (1992)

[23] H. Grabert, U. Weiss, and P. Talkner, Z. Phys. B 55, 87 (1984).

[24] W. Greiner, L. Neise, H. Stöcker Thermodynamics and Statistical Mechanics, (Springer, Berlin, 1995).

[25] M.F. Gelin and M. Thoss, Phys. Rev. E 79, 051121 (2009).

[26] J.P. Paz and A. Roncaglia, Phys. Rev. A 79, 032102 (2009).

[27] R. Dillenschneider and E. Lutz, Phys. Rev. E 80, 042101 (2009).

[28] J. Ankerhold, P. Pechukas, and H. Grabert, Phys. Rev. Lett. 87, 086802 (2001); J. Ankerhold and H. Grabert, Phys. Rev. Lett. 101, 119903 (2008).

[29] L. Machura, M. Kostur, P. Hänggi, P. Talkner, and J. Łuczka, Phys. Rev. E 70, 031107 (2004).

[30] J. Łuczka, R. Rudnicki and P. Hänggi, Physica A 351, 60 (2005).

[31] J. Ankerhold, Europhys. Lett. 61, 301 (2003).

[32] W. T. Coffey, Y. P. Kalmykov, S. V. Titov, and B. P. Mulligan, J. Phys. A. 40, F91, (2007)

[33] S.Hilt, S. Shabbir, J. Anders, and E. Lutz, Phys. Rev. E 83, 030102(R) (2011).

[34] M. Abramowitz and I.A. Stegun, Handbook of Mathematical Functions, (Dover, New York, 1972). 